\title{
Evidence of an enhanced interband absorption in Au nanoparticles: Size-dependent electronic structure and optical properties
}

\author{
AUTHOR(S): \\ Balamurugan, B; Maruyama, T
}

\section{CITATION:}

Balamurugan, B ... [et al]. Evidence of an enhanced interband absorption in Au nanoparticles: Size-dependent electronic structure and optical properties. APPLIED PHYSICS LETTERS 2005, 87(14): 143105.

\section{ISSUE DATE:}

2005-10-03

URL:

http://hdl.handle.net/2433/43531

\section{RIGHT:}

Copyright 2005 American Institute of Physics. This article may be downloaded for personal use only. Any other use requires prior permission of the author and the American Institute of Physics. 


\title{
Evidence of an enhanced interband absorption in Au nanoparticles: Size-dependent electronic structure and optical properties
}

\author{
B. Balamurugan ${ }^{\mathrm{a})}$ and Toshiro Maruyama \\ Department of Chemical Engineering, Graduate School of Engineering, Kyoto University, \\ Kyoto 615-8510, Japan
}

(Received 6 May 2005; accepted 9 August 2005; published online 27 September 2005)

\begin{abstract}
The present study reports clear evidence and physical significance of an interband transition due to the transition from the $d$ band to an empty state in the conduction band in the optical absorption spectra of Au nanoparticles. The optical absorption due to interband transitions has been observed to dominate the plasmon absorption on decreasing the particle size. X-ray photoelectron spectroscopy and optical absorption studies reveal the metallic nature even for smaller sizes of $2.5 \mathrm{~nm}$. (C) 2005 American Institute of Physics. [DOI: 10.1063/1.2077834]
\end{abstract}

An immense interest toward nanoparticle research has been growing rapidly in recent years not only due to their potential uses in technological applications, but also due to the basic physics involved with the modifications in structure and electronic redistributions of nanoparticles. These modifications ultimately lead to the size-dependent structural, optical, and electronic properties, which can be tailored to be suitable for device applications by varying the particle size. ${ }^{1-3}$ However, the purity of the nanoparticle surface, especially in case of nanoparticles prepared by chemical techniques and the postdeposition modification of the nanoparticle surface, is also expected to influence their crystallographic phase, electronic structure, and optical properties. ${ }^{4-7}$ Recently, thiol-capped $\mathrm{Au}$ nanoparticles having a particle size of $2.4 \mathrm{~nm}$ have been reported to undergo a transition from metal to insulator on the basis of an absence of the surface plasmon peak in optical absorption spectra and a clear observation of valence-band edge at zero-binding energy in the x-ray photoelectron spectroscopy (XPS) valenceband spectra. ${ }^{8}$ But, the stabilizing ligands or dispersing medium have been observed to influence the electronic structure and the shape and peak position of the surface plasmon absorption peak of $\mathrm{Au}$ nanoparticles. ${ }^{9-12}$ These results points toward the importance to investigate how the particle size influences the optical and electronic properties of $\mathrm{Au}$ nanoparticles without the influence of capping molecules or stabilizing agents. Further, it may be mentioned that earlier studies mainly focus on the effect of dispersing medium or surfactants on the optical properties of chemically prepared nanoparticles with sizes greater than $10 \mathrm{~nm}$ and does not present a systematic effect of particle size. ${ }^{10-14}$ This may be due to difficulties in distinguishing the effect of size and surfactants on the optical properties. In case of metal nanoparticles, the quantum size effects and enhanced surface effects are expected to effectively influence the optical and electronic properties at much smaller particle sizes. Thus, these results clearly lead to an increased interest to study the sole effect of particle size on the optical and electronic properties of Au nanoparticles having a pure surface without surfactants or stabilizing agents. This will be important in understanding the physical significance of size-induced optical

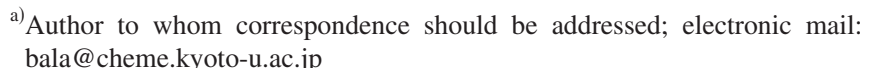

and electronic properties and implementing these nanoparticles for potential device applications.

In the present study, the structural, optical, and electronic properties of $\mathrm{Au}$ nanoparticles have been investigated. By using a radio-frequency (rf) (frequency $\sim 13.5 \mathrm{MHz}$ ) magnetron sputtering of a high-purity Au target, Au nanoparticles have been deposited on quartz substrates for optical absorption (OA) [Perkin-Elmer Lambda 19 ultraviolet-visible-nearinfrared (UV-VIS-NIR) spectrophotometer] and XPS (in which $\operatorname{Mg} K \alpha$ excitation was used) measurements and on carbon-coated copper grids for transmission electron microscopy (TEM) (JEM 2000 FX-II with an acceleration voltage $200 \mathrm{kV}$ and JEM 4000SFX with an acceleration voltage $400 \mathrm{kV}$ ) analysis. The sputtering gas used for preparing nanoparticles is highly pure argon. The deposition time has been varied as $5 \mathrm{~s}$ (Sample 1), $10 \mathrm{~s}$ (Sample 2), and $15 \mathrm{~s}$ (Sample 3) to vary the size of the Au nanoparticles in these samples while keeping the rf power, deposition pressure, flow rate of argon, and substrate temperature constant as $30 \mathrm{~W}, 120 \mathrm{mTorr}$, and $50 \mathrm{sccm}$ (standard cubic centimeters per minute) and room temperature, respectively. The purity of the Au nanoparticles has been maintained in air with out capping molecules and protective matrix.

TEM micrographs of Au nanoparticles are shown in Fig. 1. A histogram for each sample has been plotted on taking the maximum lateral width of the particles and the average particle size from the histogram has been taken as the size of the particles in the Au nanoparticle samples. The
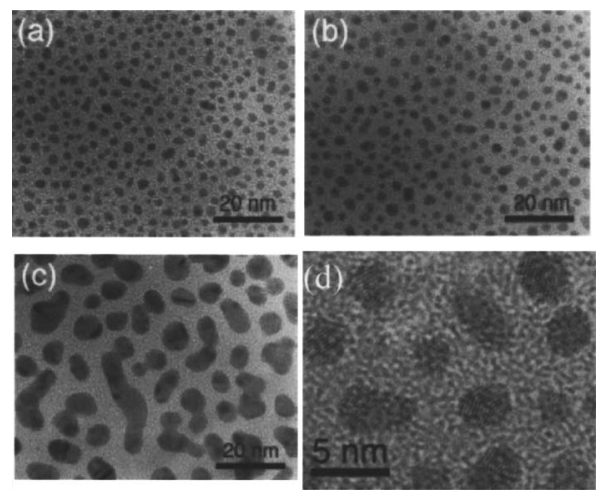

FIG. 1. TEM micrographs of Au nanoparticles: (a) Sample 1, (b) Sample 2, and (c) Sample 3. High-resolution TEM image of the nanoparticle Sample 2 is also given $[(d)]$. 


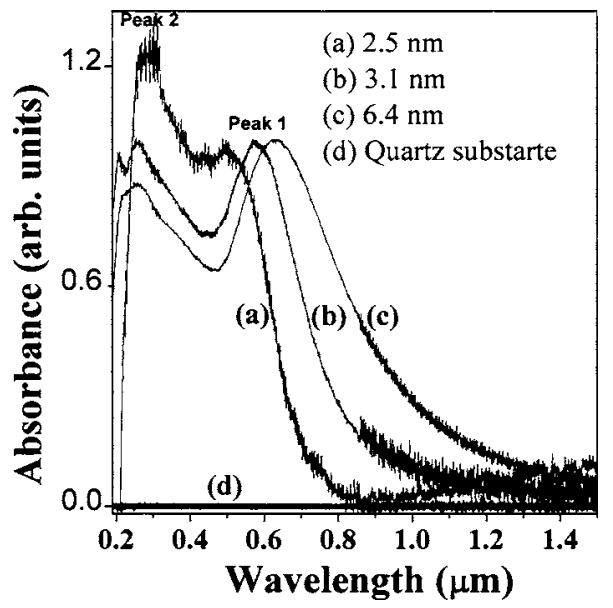

FIG. 2. OA spectra of Au nanoparticle samples (curve a-c). The absolute intensity of the plasmon peak in the $6.4 \mathrm{~nm}$ sample is two and eight times more than that of the 3.1 and $2.5 \mathrm{~nm}$ samples, respectively. To improve the clarity, the absorption spectra of Au nanoparticles (curve a-c) are normalized with the peak intensity of the plasmon absorption. The absolute intensity of the absorption spectrum of the quartz substrate showing zero absorption is also given (curve d).

average particle sizes are $2.5 \mathrm{~nm}, 3.1 \mathrm{~nm}$, and $6.4 \mathrm{~nm}$, respectively, in Sample 1 [Fig. 1(a)], Sample 2 [Fig. 1(b)], and Sample 3 [Fig. 1(c)], respectively. An increase in the sputtering time is known to increase the nucleation and growth of the particles on the substrates and thus, results in an increase in the particle size. ${ }^{15}$ Au nanoparticles also shows good crystalline nature and pure surface without any oxidation and structural changes as shown in the high-resolution TEM image of Au nanoparticle Sample 2 [Fig. 1(d)].

Figure 2 (curve a-c) shows the OA spectra of Au nanoparticles deposited on quartz substrates. Prior to absorption measurements on the Au nanoparticle samples, the background correction was performed using two identical and bare quartz substrates (as used for deposition of nanoparticles) as a reference and sample, respectively. With this background correction, the OA spectra of $\mathrm{Au}$ nanoparticle samples were recorded using one bare quartz substrate as a reference. The quartz substrate is known to be completely transparent throughout the UV-VIS-NIR wavelength region and the recorded absorbance spectrum of the bare quartz substrate (curve d in Fig. 2) in the present study also reveals zero absorption throughout the spectral range used in this study. With no contribution of quartz substrate to the optical absorption of the Au nanoparticle samples deposited on identical quartz substrates, the changes in the optical absorption spectra with a decrease in the nanoparticle size can be taken as a sole effect of the particle size. As shown in Fig. 2, the OA spectra of Au nanoparticle samples consist of two peaks situated in the visible (labeled as Peak 1) and ultraviolet (labeled as Peak 2) wavelength regions, respectively. The Peak 1 appeared in the visible wavelength region can be attributed to the plasmon peak, which is the characteristic absorption of metals. ${ }^{16}$ It is also worthwhile to note that the plasmon peak shifts from $634 \mathrm{~nm}$ to $492 \mathrm{~nm}$ with the decrease in the particle size from $6.4 \mathrm{~nm}$ to $2.5 \mathrm{~nm}$. The observed blueshift in the peak position of plasmon absorption can be attributed to the quantum size effects. ${ }^{17}$

The observation of an additional absorption peak in the ultraviolet wavelength region (Peak 2) is the important result of the present study. Peak 2 can be attributed to the absorpDownloaded 19 Jun 2007 to 130.54 .110 .22 . Redistribution subject tion due to the interband transition in Au nanoparticles. An interband absorption can be possible at shorter wavelengths due to the transition of an electron from the occupied $d$-level state to an empty state in the conduction band above the Fermi level in noble metals. ${ }^{18-21}$ A weak and broad absorption peak corresponding to interband transition has been observed at a wavelength of around 240-320 nm with a very strong plasmon peak in chemically prepared $\mathrm{Au}$ and $\mathrm{Ag}$ nanoparticles. ${ }^{18,19}$ The surfactants and capping molecules have also been observed to influence the intensity and width of the interband absorption peak and this phenomenon has been successfully used for biosensor applications. ${ }^{18}$ In addition, theoretical investigations also show a considerable contribution of interband transition to the absorption spectra of $\mathrm{Au}$ and $\mathrm{Ag}$ nanoparticles at shorter wavelengths. ${ }^{22}$ With the absence of surfactants or capping molecules, the interband absorption peak in the present study is more intense and narrow and its intensity also increases with decrease in the particle size and completely dominates against the plasmon peak at a particle size of $2.5 \mathrm{~nm}$. In addition, a sharp drop of its intensity (Peak 2) around $0.2 \mu$ along with the shift of its peak position and the wavelength at which sudden drops occurs, toward higher wavelength, have been observed with decrease in the nanoparticle size to $2.5 \mathrm{~nm}$. In nanoparticles, the electronic energy levels are not continuous as in bulk materials, but discrete due to the quantum size effects of the careers and this can result in a sharp and an intense absorption features corresponding to interband transitions in nanoparticles. $^{23,24}$ The transition from the $5 d$ band having an enormously higher density of states to the unoccupied discrete energy level in the conduction band is expected to be flat and this leads to a steplike structure having a sudden drop. ${ }^{24}$ With decrease in the nanoparticle size, the discrete nature of the bands increases with an increase in the gap known as the Kubo gap between the discrete levels, which can result in a shift of the first unoccupied discrete level toward the $d$ band. $^{23,24}$ This can be the possible reason for the shift in the peak position of Peak 2 and the wavelength at which sudden drops occur toward the higher wavelength side.

The presence of plasmon peak in the absorption spectra also reveals the metallic nature of $\mathrm{Au}$ nanoparticles. To observe direct evidence for metallic nature, valence-band XPS spectra have been recorded on the Au nanoparticle samples (Fig. 3). The zero point in the energy scale in Fig. 3 corresponds to the Fermi-level position $\left(E_{F}\right)$ and two predominant broad peaks centered at around $3 \mathrm{eV}$ and $6 \mathrm{eV}$ corresponds to the contribution of $\mathrm{Au} 5 d$ electrons to the electronic structure of Au. ${ }^{25}$ The electronic states below $E_{F}$ clearly reveal the metallic nature of $\mathrm{Au}$ nanoparticles. In addition, the peak position of the recorded XPS $4 f$ core-level spectra recorded on the vicinity of the surface of the Au nanoparticles (Fig. 4) does not show any major chemical shift with respect to that of reported value of elemental $\mathrm{Au},{ }^{26}$ which reveals the purity of the Au nanoparticle surface. However, a subtle increase of $0.1 \mathrm{eV}$ in the XPS core-level spectra and the width of the XPS valence-band spectra on decreasing particle size observed in the present study can be attributed due to the sizeinduced electronic redistributions and a strong Coulombic interaction between the core hole and outgoing photoelectron. ${ }^{23,25,27}$ In contrast to the metallic nature of Au nanoparticles observed in the present study, thiol-capped Au nanoparticles have been observed to undergo a metal to into AIP license or copyright, see http://apl.aip.org/apl/copyright.jsp 


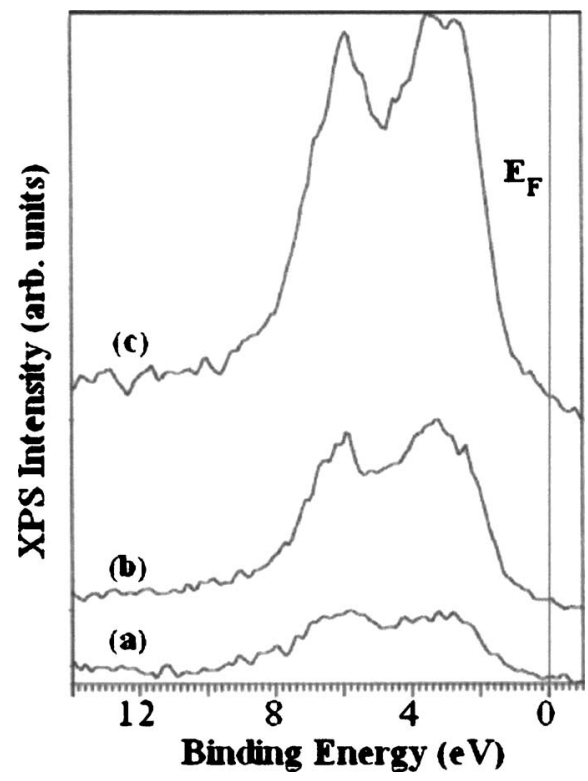

FIG. 3. Valence-band XPS spectra of Au nanoparticle samples having different average particle sizes: (a) $2.5 \mathrm{~nm}$, (b) $3.1 \mathrm{~nm}$, and (c) $6.4 \mathrm{~nm}$.

sulator transition at a particle size of $2.4 \mathrm{~nm} .{ }^{8}$ But, the surfactants and capping molecules can also influence the optical and electronic properties of the nanoparticles. In support to the present study, Au nanoparticles of size $1.6 \mathrm{~nm}$, stabilized by chemical ligands, show an insulating nature due to the strong intact ligand shells with the nanoparticles and becomes metallic on the removal of the chemical ligands by x-ray exposure. ${ }^{9}$ Thus, the present study reveals the importance of the purity of the nanoparticle surface to observe the size-induced changes in optical and electronic properties of nanoparticles.

In conclusions, OA spectra of Au nanoparticles prepared by rf magnetron sputtering shows a clear, dominant, and discrete absorption peak due to interband transitions along with the plasmon peak. The interband absorption dominates the

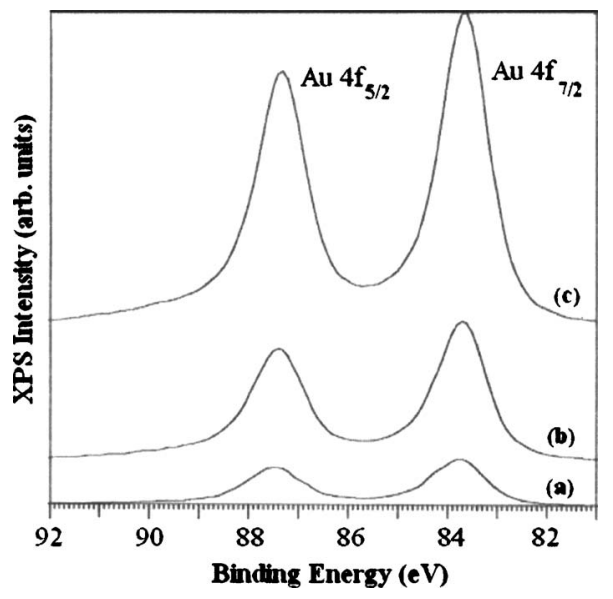

FIG. 4. XPS spectra of Au $4 f_{7 / 2}$ and $\mathrm{Au} 4 f_{5 / 2}$ core electrons of Au nanoparticle samples having different average particle sizes: (a) $2.5 \mathrm{~nm}$, (b) $3.1 \mathrm{~nm}$, and (c) $6.4 \mathrm{~nm}$. plasmon absorption on decreasing particle size. The observation of electronic states below the Fermi-level position together with the metallic characteristic plasmon peak in the absorption spectra clearly reveal the metallic nature of the $\mathrm{Au}$ nanoparticles. This study also explains how the contamination-free nanoparticle surface is essential to observe the size-dependent optical and electronic properties.

One of the authors (B.B.) acknowledges the financial support from Japan Society for the Promotion of Science (JSPS). A part of this work was supported by the Nanotechnology Support Project of the MEXT Japan.

${ }^{1}$ K. Koga, T. Ikeshoji, and K. Sugawaral, Phys. Rev. Lett. 92, 115507 (2004).

${ }^{2}$ Z. S. Li, C. X. Kan, and W. P. Cai, Appl. Phys. Lett. 82, 1392 (2003).

${ }^{3}$ P. M. Paulus, A. Goossens, R. C. Thiel, A. M. van der Kraan, G. Schmid, and L. J. de Jongh, Phys. Rev. B 64, 205418 (2001).

${ }^{4}$ N. Herron, J. C. Calabrese, W. E. Farneth, and Y. Wang, Science 259, 1426 (1993).

${ }^{5}$ R. Antonine, M. Pellarin, B. Palpant, B. Prevel, P. Galletto, P. F. Brevet, and H. H. Girault, J. Appl. Phys. 84, 4532 (1998).

${ }^{6}$ B. Balamurugan, B. R. Mehta, and S. M. Shivaprasad, Appl. Phys. Lett. 79, 3176 (2001)

${ }^{7}$ C. Prados, M. Multigner, A. Hernando, J. C. Sanchez, A. Fernandez, C. F. Conde, and A. Conde, J. Appl. Phys. 85, 6118 (1999).

${ }^{8}$ P. Zhang and T. K. Sham, Phys. Rev. Lett. 90, 245502 (2003).

${ }^{9}$ H. G. Boyen, G. Kästle, F. Weigl, P. Ziemann, G. Schimid, M. G. Garnier, and P. Oelhafen, Phys. Rev. Lett. 87, 276401 (2001).

${ }^{10}$ S. Huang, K. Minami, H. Sakaue, S. Shingubara, and T. Takahagi, J. Appl. Phys. 92, 7486 (2002).

${ }^{11}$ C. D. Gedds, A. Parfenov, I. Gryczynski, J. R. Lakowicz, Chem. Phys. Lett. 269, 380 (2003).

${ }^{12}$ S. K. Gosh, S. Nath, S. Kunda, K. Esumi, and T. Pal, J. Phys. Chem. B 108, 13963 (2004).

${ }^{13}$ W. Rechberger, A. Hohenau, A. Leitner, J. R. Krenn, B. Lamprecht, and F. R. Aussenegg, Opt. Commun. 220, 137 (2003).

${ }^{14}$ D. M. Schaadt, B. Feng, and E. T. Yub, Appl. Phys. Lett. 86, 063106 (2005).

${ }^{15}$ M. Hirasawa, H. Shirakawa, H. Hamamura, Y. Egashira, and H. Komiyama, J. Appl. Phys. 82, 1404 (1997).

${ }^{16}$ F. Wooten, Optical Properties of Solids (Academic, New York, 1972).

${ }^{17}$ H. S. Zhou, I. Honma, H. Komiyama, and J. W. Haus, Phys. Rev. B 50, 12052 (1994).

${ }^{18}$ F. Frederix, J. M. Friedt, K. H. Choi, A. Campitelli, D. Mondelaers, G. Maes, and G. Borghs, Anal. Chem. 75, 6894 (2003).

${ }^{19}$ M. M. Alvarez, J. T. Khoury, T. G. Schaaff, M. N. Shafigullin, I. Vezmar, and R. L. Whetten, J. Phys. Chem. B 101, 3706 (1997).

${ }^{20}$ C. Voisin, D. Christofilos, N. D. Fatti, F. Vallee, B. Prevel, E. Coottancin, J. Lerme, M. Pellarin, and M. Broyer, Phys. Rev. Lett. 85, 2200 (2000).

${ }^{21}$ P. P. Kiran, B. N. S. Bhaktha, D. N. Rao, and D. Goutam, J. Appl. Phys. 96, 6717 (2004).

${ }^{22}$ A. Pinchuk, G. V. Plessen, and U. Kreibig, J. Phys. D 37, 3133 (2004).

${ }^{23}$ C. N. R. Rao, G. U. Kulkarni, P. J. Thomas, and P. P. Edwards, Chem. A Euro. J. 8, 28 (2002).

${ }^{24}$ T. G. Schaaff, M. N. Shafigullin, J. T. Kohoury, I. Vegmar, R. L. Whetten, W. G. Cullen, P. N. First, C. G. Wing, J. Ascensio, and M. J. JoseYacaman, J. Phys. Chem. B 101, 7885 (1997).

${ }^{25}$ M. G. Boyen, A. Ethirajan, G. Kästle, F. Weigl, P. Ziemann, G. Schimid, M. G. Garnier, M. Buttner, and P. Oelhafen, Phys. Rev. Lett. 94, 016804 (2005).

${ }^{26}$ J. Hedman, M. Klasson, R. Nilsson, C. Nordling, M. F. Sorokina, O. I. Kljushnikov, S. A. Nemnonov, V. A. Trapeznikov, and V. G. Zyranov, Phys. Scr. 4, 195 (1971).

${ }^{27}$ P. Moriarity, Phys. Rev. Lett. 92, 109601 (2004). 